Revista de Artes Marciales Asiáticas

Volumen $10 \sim 2015$ / Volume $10 \sim 2015$ / Volume 10 2015

\title{
Revisores / Reviewers
}

- Ildefonso Alvear Órdenes (Universidad de León, España).

- Paulo Coelho Araújo (Universidade de Coimbra, Portugal).

- Bruno Avelar Rosa (Universidade Europeia de Lisboa, Portugal).

- Ciro Brito (Federal University of Juiz de Fora, Brasil).

- Raquel Escobar Molina (Universidad de Granada, España).

- Isaac Estevan (Universitat de Valéncia, España).

- Ursula Ferreira Julio (Universidade de São Paulo, Brasil).

- Emerson Franchini (Universidade de São Paulo, Brasil).

- Thomas A. Green (Texas A\&M University, United States).

- Carlos Gutiérrez García (Universidad de León, España).

- Ana Rosa Jaqueira (Universidade de Coimbra, Portugal).

- José Luis López Elvira (Universidad Miguel Hernández de Elche, España).

- Javier Eloy Martínez Guirao (Universidad de Murcia, España).

- Bianka Miarka (Universidade de São Paulo, Brasil).

- Bill Newman (State University Plovdiv, Bulgaria).

- Artur Manuel Romão Pereira (Universidade de Coimbra, Portugal).

- Mikel Pérez Gutiérrez (Universidad Autónoma de Chile, Chile).

- Elena Pocceco (University of Innsbruck, Austria).

- Zdenko Reguli (Masaryk University, the Czech Republic).

- Vitor Rosa (Université Paris Ouest Nanterre La Défense, France)

- Roberto Ruiz Barquín (Universidad Autónoma de Madrid, España).

- Raúl Sánchez García (Universidad Europea de Madrid, España).

- Juliano Schwartz (Universidade de São Paulo, Brasil).

- Denys Toichkin (Institute of History of Ukraine, Ukraine).

- Daniel Giordani Vasques (Universidade Federal do Recôncavo da Bahia, Brasil).

- Michal Vit (Masaryk University, the Czech Republic). 